\title{
Los acuerdos de paz y la democratización
}

\author{
Héctor Dada Hirezi
}

\begin{abstract}
Resumen
Testimonio del proceso salvadoreño de alguien que ha luchado largamente por la democracia con justicia social. El autor defiende que sólo la lucha social favorece la distribución en contraposición a quienes atribuyen esta función al mercado. No obstante algunos obstáculos graves para la participación y el debate, ya existen signos que apuntan hacia una mayor democratización del país. Sin embargo, para llegar a ella será necesario resolver los graves problemas de exclusión y marginación del país. El autor leyó esta ponencia en el Auditorio de la Universidad Centroamericana "José Simeón Cañas", el 23 de abril de 1995.
\end{abstract}

Como fui invitado a participar en esta mesa redonda acepté casi de inmediato, dado mi interés por la historia y por el tema del Estado. Después tuve serias dudas, que procesé con los organizadores: ¿qué aportes podía dar siendo ajeno profesionalmente a la historia, a la sociología y a la ciencia política? Y la respuesta que se me dio me impulsó a aceptar el reto: lo que se me solicitaba era una presentación testimonial, una visión de ciudadano, una reflexión de más de tres decenios de hacer política, dos de los cuales en forma partidaria y los últimos quince años desde la trinchera de la mayoría de las personas: la ciudadanía por lograr.

Por ello no debe esperarse un trabajo académico en el sentido noble del término. Ni aportes teóricos, ni análisis dentro de la metodología histórica, ni predicciones del futuro. Es eso, un testimonio, testimonio de un proceso, y por ello -no sólo por estar entre historiadores- dedico un espacio a recordar las raíces de nuestra transición actual.

Muchos de los participantes estoy seguro que puede enriquecer mucho más que yo este tema. Eso es un aporte muy sustancial que requiere el país. El salvadoreño es muy adicto a pretender el olvido de la historia. Además de ser imposible, es dañino, pues impide conocer las causas de lo que se quiere expresar, el proceso en el que se puede superar y la supervivencia de lo viejo que obstaculiza la superación. Por ello, eventos como éste tienen una trascendencia que supera a lo estrictamente académico.

\section{Características del modelo político hasta 19791}

Es común afirmar que El Salvador ha vivido 


\section{Esta permanente contradicción entre el derecho y la realidad exigió siempre garantizar institucionalmente la impunidad a quienes ejercían el poder.}

tradicionalmente un régimen autoritario y excluyente. Estas características son comunes a las que presentan otros países del continente, pero parece conveniente relatar muy someramente cómo se expresa en la realidad concreta. En primer lugar, es necesario decir que el régimen autoritario fue una necesidad inherente al modelo económico social del país. Si bien suele simplificarse el análisis, atribuyendo exclusivamente a la mala voluntad de los militares los rasgos autoritarios de la forma de gobierno -por lo que algunos centran en el cambio de la mentalidad de los militares, en su profesionalización y en su "democratización" la base de la transformación democrática-, la verdad es que el predominio de la burocracia militar en las decisiones políticas surge de la forma de relaciones económicas y sociales que genera un modelo agroexportador absolutamente excluyente, y de la incapacidad de la burocracia civil para asumir la conducción del gobierno.

Sin embargo, la realidad autoritaria coincide con una legislación que consagra los principios democráticos. Las constituciones de 1950 y 1961 fueron vistas por muchos analistas como textos constitucionales de avanzada, que llegaban incluso a reconocer el derecho del pueblo a la insurrección contra el autoritarismo; si bien establecían restricciones a los "partidos internacionales", contenían todos los elementos jurídicos para garantizar los derechos políticos fundamentales de una democracia. Esta coincidencia de realidad autoritaria y legislación democrática genera una forma muy pervertida del ejercicio del poder: tiene necesidad de impedir que la obligación de votar y el derecho de elegir, garantizados tanto en la Constitución como en la legislación secundaria, sean realmente ejercidos por la población. Para evitar que este derecho legal a la participación política -en un país donde el voto es obligatorio- se convirtiera en capacidad para crear alternativas, para prevenir que el derecho de organización resultara en una construcción de poder capaz de disputar la participación en los beneficios del desarrollo, el Estado debía violar las leyes que él mismo había establecido.

Esta permanente contradicción entre el derecho y la realidad exigió siempre garantizar institucionalmente la impunidad a quienes ejercían el poder. Esta impunidad, lejos de ser una aberración, fruto de voluntades individuales, es un elemento intrínseco al modelo. Ella convirtió al órgano judicial en un instrumento fundamental para ejercer el poder autoritario.

En este contexto, las elecciones son sólo poco más que una ceremonia litúrgica, en la cual los ciudadanos deben legalizar un gobierno previamente escogido por una fracción de la burocracia del Estado, las fuerzas armadas. Debe decirse que la percepción de fraude electoral no alteraba fundamentalmente este hecho, pues en definitiva, no se cuestionaba el derecho de escogitación que de forma pasiva y a veces activa se le reconocía a la burocracia militar. Aunque parezca paradójico, la prueba mayor de este derecho no escrito a determinar el resultado electoral es el hecho de que las mismas fuerzas políticas más que apelar a los órganos judiciales para exigir el reconocimiento de sus triunfos frente a los fraudes reales o supuestos, lo hacían a la conciencia de la Fuerza Armada y a su deber de hacer respetar la Constitución.

A pesar de diversos intentos para modernizar y abrir el régimen político ("democratización", se decía), las más de las veces, bajo presiones externas como las de la Alianza para el Progreso, el sistema excluyente y autoritario continuó vigente, si bien se generaron espacios para la participación opositora siempre que no pusieran en peligro la lógica fundamental de su funcionamiento. Pese a las grandes movilizaciones populares, a la alta dosis de participación electoral en los años sesenta y a principios de los setenta, que reflejaba una expectativa de cambio por las vías legales, pronto los límites de la apertura quedaron a la luz del día y el recrudecimiento de la represión no se hizo 
esperar. En un momento de gran optimismo sobre el futuro, en el que la disputa no era si se iba a crecer, sino cómo se iba a crecer y a favor de quién, en el que la responsabilidad del Estado de generar justicia social no era discutida, en el que se planteaba el dilema de la "democracia burguesa" y la "democracia popular", los espacios políticos volvieron a cerrarse. Y para un buen grupo de jóvenes, la mayoría de los cuales había participado en la lucha por la democracia a través de las elecciones, la lucha armada pareció la mejor forma de tomar la justicia por la propia mano. Permítaseme citar al ex presidente Alfredo Cristiani, que en su discurso en el Castillo de Chapultepec, al firmar los acuerdos de paz, dijo: "La crisis en que se vio envuelta la sociedad salvadoreña en el último decenio no surgió de la nada, ni fue producto de voluntades aisladas. Esta crisis tan dolorosa y trágica tiene antiguas y profundas raíces sociales, políticas, económicas y culturales, [en] la inexistencia o insuficiencia de los espacios y mecanismos necesarios para permitir el libre juego de las ideas... en síntesis, la ausencia de un verdadero esquema democrático de vida".

\section{De la crisis a la guerra}

El modelo hizo crisis a partir de finales de los años setenta, al perder sus bases materiales y sus bases políticas. La oligarquía tradicional, de base fundamentalmente agroexportadora, principal beneficiaria del sistema, perdió su capacidad para controlar el proceso económico, primordialmente a causa de la crisis en que había entrado la economía internacional. Esta situación, que no es del caso describir con mayor detalle, privó también al Estado de los recursos necesarios para cumplir su tarea de sostén del proceso de sustitución de las importaciones.

Desde el punto de vista de la gobernabilidad, éste ya era un elemento que la debilitaba. Una presencia permanente de las masas en concentraciones, huelgas, movilizaciones, etc., que no podían ser impedidas ni canalizadas por el gobierno a pesar de las altas dosis de violencia utilizadas en su contra, hizo que en todo el período que presidió el general Romero (1977-1979), la ingobernabilidad fuera casi total. La caída de la dictadura nicara-

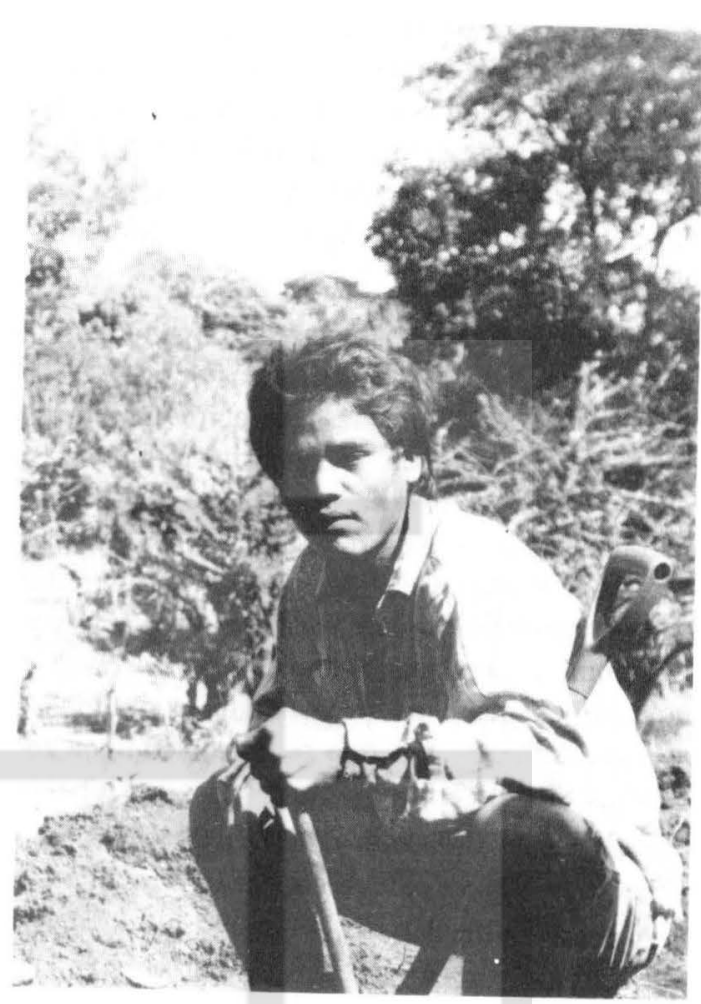

güense contribuyó a profundizar la problemática.

En octubre de 1979, la Fuerza Armada dio un golpe de Estado que terminó de desintegrar la alianza de poder del viejo modelo autoritario e intentó sustituirla por una nueva, mucho más concertadora, negociando la inclusión de las fuerzas políticas y sociales hasta entonces marginadas. Realidades externas, pero primordialmente la dinámica interna, hicieron que todos los esfuerzos hechos en esos momentos para encontrar una forma no violenta para transformar la realidad politica del país fracasaran completamente. La dialéctica insurgencia-contrainsurgencia dominó el escenario hasta la firma de los acuerdos de paz.

La contrainsurgencia adquirio una característica que conviene resaltar: la internalización de los agentes externos. Estos agentes, muchas veces con una larga experiencia en la orientación de la politica salvadoreña, perdieron en buena medida su carácter externo para convertirse en verdaderos elementos del poder interno, factores centrales en la generación de alianzas de poder, así como determinantes fundamentales de las políticas economi- 
ca y militar. Esto implicó una reducción apreciable del rol de las fuerzas armadas en la decisión sobre quién y cómo se debe gobernar el país. A eso hay que agregar una enorme cantidad de recursos para sostener militar y financieramente todo el conjunto de políticas para contener la insurgencia.

Si bien no puede hablarse de la generación de espacios o relaciones democráticas durante la guerra, sí puede constatarse la generación de mecanismos de cierta competencia política relativamente equitativa entre los partidos que apoyaban la contrainsurgencia. Reformas económicas y sociales, guerra y elecciones parecen ser los ingredientes de un programa que buscaba aislar social y políticamente a las organizaciones guerrilleras y generar la sensación de una inédita posibilidad de participación, a la vez que se enfrentaba con toda la fuerza posible a la organización de los rebeldes, tanto por la guerra como por la más cruda represión.

A pesar de los esfuerzos y los recursos puestos en juego, las partes implicadas en el conflicto, lejos de lograr la derrota del contrario, llegaron a lo que diversos analistas han llamado una "situación de vetos simultáneos": la extrema derecha impide que el reformismo del programa contrainsurgente se desarrolle con toda su amplitud; la contrainsurgencia no permite ni el triunfo revolucionario ni la restitución del poder oligárquico; la izquierda quita toda posibilidad de consolidación al proyecto contrainsurgente, a la vez que bloquea los intentos para reconstituir el autoritarismo del pasado. En síntesis, una situación donde la "solución" por la vía militar es imposible, al mismo tiempò que se da un empantanamiento en el plano político.

Los cambios en el orden internacional tuvieron un peso considerable en la evolución del conflicto, no sólo por la limitación del ejercicio de la soberanía, resultado de la presencia de los actores extranjeros, sino por la misma naturaleza de esas transformaciones. La crisis del "socialismo real" y el agotamiento del proceso revolucionario en Nicaragua, entre otras razones, hicieron desaparecer referentes ideológicos en las fuerzas insurgentes, que algunos analistas han llegado a señalar como la causa del abandono del objetivo del triunfo revo- lucionario. El fin de la "guerra fría" minó el soporte político militar que Estados Unidos proporcionaba a la guerra de contrainsurgencia y fue volviendo intolerables los costos de una victoria militar, a la vez que la desaparición de las contradicciones irreconciliables entre las fuerzas políticas tornaron posible la incorporación de las fuerzas insurgentes _ya desarmadas_al sistema político. Estos factores que mencionamos muy someramente, a riesgo de caricaturizarlos, se interrelacionan con el reclamo de la mayoría de la población para poner término al conflicto, dada la poca perspectiva de la salida militar a corto plazo. Negociar la paz se volvió una necesidad imperiosa, y el diálogo y la negociación -por un buen tiempo elementos tácticos de la estrategia de la guerra- se convirtieron en elementos estratégicos para la construcción de la paz.

\section{Los acuerdos de paz de Chapultepec}

El proceso de negociación hizo un largo y tortuoso camino desde que José Napoleón Duarte, poco después de tomar posesión de la presidencia en 1984, llamó al diálogo, aprovechando su presencia en la asamblea general de Naciones Unidas. Con avances y retrocesos, encabezado el gobierno salvadoreño por quienes al principio proclamaban que negociar era traicionar al país, la negociación entró en su cauce definitivo en el gobierno del presidente Cristiani. Esta tiene dos grupos de actores, llamados por los diversos documentos "los contendientes": por un lado, el gobierno y la Fuerza Armada (a los cuales hay que agregar el gobierno de Estados Unidos por su nivel de participación en los aspectos militares y políticos del conflicto) $y$, por el otro lado, las cinco organizaciones político militares que conformaban el FMLN, acompañadas por los partidos políticos aliados (incorporados al Frente Democrático Revolucionario) y las fuerzas sociales afines.

La presencia de fuerzas externas, tanto en las interioridades de la negociación como en las acciones directas sobre los actores afuera de la mesa, fue un elemento central para viabilizar el proceso. Naciones Unidas, con su Secretario General a la cabeza, se convirtió en la mediadora y garante oficial de los acuerdos que fueron surgiendo de la 
prolongada serie de conversaciones.

Los acuerdos de paz -que para muchos actores y estudiosos del proceso son un "viraje histórico"- se proclamaron como un punto de partida para la construcción de una sociedad respetuosa de las diferencias entre sus componentes, rompiendo con la tradicional cultura del autoritarismo. Desmontando programadamente $-y$ a partir de un pacto entre los contendientes en el conflicto armado- las formas institucionalizadas del régimen de exclusión política se dio paso a la posibilidad de participación pluralista de todas las fuerzas políticas. Asimismo, se adquirió el compromiso de impulsar la superación de la situación de dependencia y exclusión a la que históricamente se sometía a la mayoría de la población.

Los signatarios de los acuerdos lo expresaron con gran optimismo el 16 de enero de 1992, en el Castillo de Chapultepec. El presidente Alfredo Cristiani dijo que "la crisis hizo surgir la posibilidad real de la democracia en nuestro país... la esperanza [de todo un pueblo] en una vida más humana y más plena, sin exclusiones y privilegios, toma cuerpo en el Acuerdo de paz y por ello decimos que este Acuerdo es una plataforma de armonía para el presente y para el futuro". Para Schafick Handal, entonces secretario general del Partido Comunista Salvadoreño, "los acuerdos que hemos firmado contienen el diseño del nuevo país que deseamos los salvadoreños, de la vida que queremos vivir"2.

En el proceso de negociación se establecieron reformas a las leyes secundarias y a la misma Constitución, como parte de la búsqueda de garantía para el funcionamiento de la democracia política y para la seguridad de la integración del FMLN al sistema político y de sus combatientes a la vida civil. Si la razón para alzarse en armas había sido la injusticia social, ahora la explicación del desarme era la inclusión en la lucha política legal para la construcción de la democracia. Esto explica la acuciosidad con la cual se discutieron las reformas legales e institucionales que permitieron una competencia relativamente equitativa y que desmontaron los engranajes fundamentales del autoritarismo.
La parte económico social de los acuerdos es mucho más limitada. No atacan -y no podían hacerlo, pues no era posible que se hicieran cargo de toda problemática nacional- las causas fundamentales que generan la exclusión social y económica ni se discuten los ejes fundamentales de la economía de mercado que aplica el gobiemo de ARENA. Si bien se establecieron medidas para distribuir tierras - por otro lado permitidas por el colapso anterior del modelo agrario exportador-, eso se hizo en beneficio de los ex combatientes de ambos ejércitos y para facilitar su tránsito de elementos armados a civiles. David Escobar Galindo, miembro de la comisión negociadora gubernamental, lo expresó claramente en un foro organizado por FLACSO:

El sistema político salvadoreño hizo crisis profunda y nos llevó a la guerra. Por consiguiente, si queríamos resolver la guerra por la vía de un acuerdo negociado, este debía ser de naturaleza política. Y por eso en cuanto algunas personas

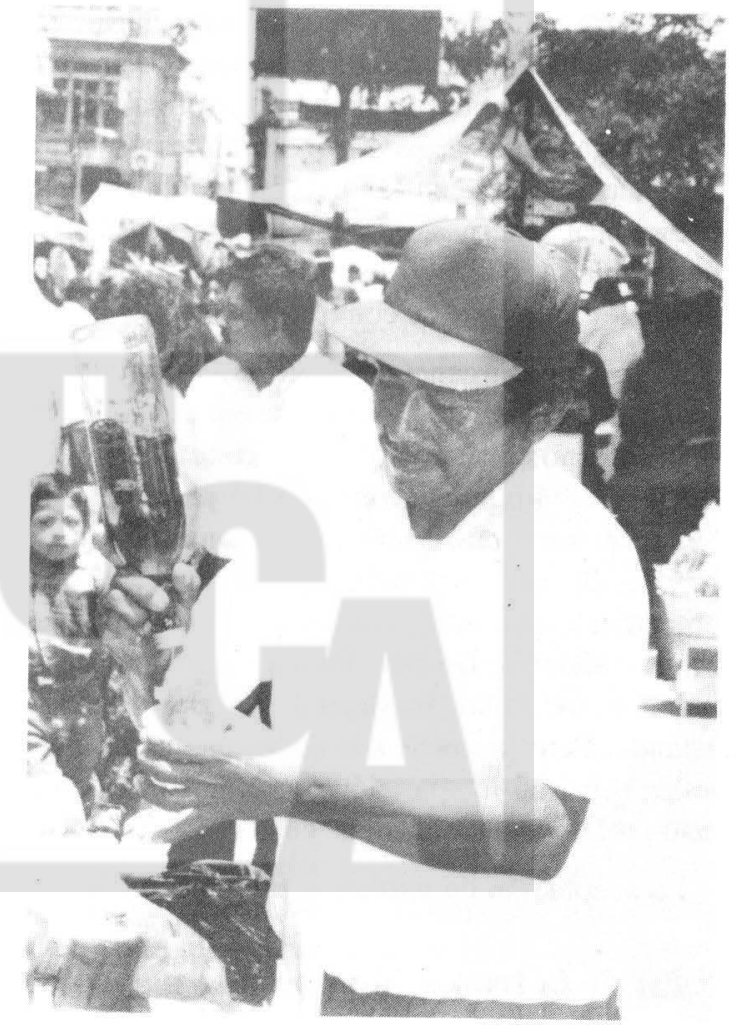


dicen que ese acuerdo sólo es político y que por qué no resolvió el problema económico y social, yo siempre pienso que hubiera sido un desgaste inútil estar tratando de hacer un modelo económico-social en el acuerdo. Lo que se tenía que hacer, y fue lo que se hizo, es sentar las bases políticas para que las diversas fuerzas del país con distintos grupos ideológicos, distintas maneras de percibir la realidad, pudieran plantearle a quien se le debe plantear eso -al electorado, al pueblo votante-, las distintas opciones sociales y económicas para que decidan. Porque si no ¿qué hubieramos hecho en la mesa?; ¿el proyecto de quién?; ¿un híbrido?3.

\section{La transición}

La crisis del ejercicio autoritario del poder no comienza con el fín de la guerra. Es más, en cierta manera, la guerra - la dimensión que adquirí́ y las condiciones en que se desarrollo- es debida o al menos permitida por el agotamiento de la capacidad para gobernar del bloque autoritario. La ingobernabilidad casi total que se manifiesta a finales de los setenta no es sino la etapa final de una crisis largamente gestada por múltiples factores. La radicalidad del conflicto social y político y las condiciones externas orientaron el proceso a la guerra abierta, evitando una transición "ordenada" hacia nuevas formas de convivencia social.

La contrainsurgencia conducida de modo preferente por los estadounidenses - pero con bloques internos aliados parcial o totalmente a ellos- produjo, por su misma percepción del problema, la coexistencia del esfuerzo militar con la apertura de espacios para la participación y la competencia electoral para los partidos aceptables al nuevo sistema. Un embrión de sistema de partidos -con todas las limitaciones lógicas- se fue gestando. Pero la frecuencia de los ejercicios electorales permitió la aparición de formas de participación relativamente inéditas en el país.

La aceptación de participar en los comicios de
1989 a condición de su posposición de acuerdo a los términos de la Constitución vigente fue un hito en el proceso de negociación 4 . Por primera vez en un decenio, las fuerzas agrupadas en el FMLN aceptaron la legitimidad de un orden juridico y la validez del proceso electoral como mecanismo para disputar el gobierno. Si bien la guerra continuó, ésta se planteó como un instrumento de presión para llegar a una paz democrática.

Si este hecho, y otros que no tenemos espacio para señalar aquí, aceleraron la negociación, los acuerdos de paz son evidentemente un hecho histórico. Explicados y facilitados por los procesos internos y externos, éstos no pueden minimizar la importancia que tienen como momento en el cual se pacta una transición a la democracia prácticamente calendarizada. La transformación político institucional del país se aceleró y abrió espacios para la convivencia y la participación. Si bien firmados por los "contendientes" ya descritos anteriormente, los acuerdos representan el resultado de, y un nuevo impulso a, las reconversiones conceptuales y conductuales de la sociedad salvadoreña, y plantean retos inéditos para la "reconstrucción" nacional.

Lo que pudiéramos denominar "transición pactada" fue prácticamente concluida con las elecciones generales de marzo y abril de 1994. No ignoramos que muchos de los compromisos incluidos en el documento suscrito en Chapultepec no han sido cumplidos en su totalidad, y que algunos sólo han sido respetados de manera formal. Nuestra afirmación parte del hecho de que los objetivos básicos del proceso de paz han sido cumplidos, y que los suscriptores de los Acuerdos se han transformado lo suficiente como para no seguir siendo "las partes" a ser exigidas por lo no obtenido en forma y fondo. La institucionalización de buena parte de las reformas, la inclusión del FMLN en el sistema de partidos, la aceptación de legitimidad de los resultados electorales están allí para corroborar lo que afirmamos.

Sin embargo, la democracia no está lograda.

\section{El fin de la transición pactada nos coloca a las puertas de una nueva etapa de lucha social y política, muy distinta a la del pasado reciente.}


Los retos no han sido respondidos. En cierta manera, el fin de la transición pactada significa entrar de lleno a otro proceso de transición, no menos acelerado, que va a definir realmente el tipo de sociedad que va a construirse en El Salvador, si ésta va a ser de matriz preferentemente autoritaria o si será de matriz preferentemente democrática. Entre 1992 y 1994 se dio un cambio de régimen aún no consolidado; asimismo, se inició un reacomodo de las relaciones de poder al interior de las clases dominantes y entre éstas y las clases subalternas. El fin de la transición pactada nos coloca a las puertas de una nueva etapa de lucha social y política, muy distinta a la del pasado reciente.

\section{Los retos de la construcción democrática}

Como hemos dicho, la negociación abrió espacios para una transición hacia formas más democráticas de convivencia nacional. Pero si va a llevarnos realmente a la democracia es, hoy por hoy, sólo una responsabilidad. Cierto que no hay reversiones posibles: no hay condiciones objetivas -aunque sobre quien lo sueñe para retornar al autoritarismo del pasado, como tampoco las existen para reeditar la guerra tal como la vivimos. Lo que sí existe es la posibilidad real de un autoritarismo de nuevo cuño, una nueva visión de inclusiones y exclusiones a partir de la conversión de los intereses de un sector en los de la sociedad en su conjunto.

Si caminamos hacia la democracia, tenemos que comenzar por convencernos que ella es fundamentalmente el reconocimiento -a todos y a cada uno de los miembros de la sociedad- del derecho de participar en la definición del destino del país, en la selección de quiénes van a conducir el Estado y en la decisión de las políticas a ejecutar. Esto supone la aceptación de que los miembros de la sociedad son diferentes, piensan distinto, tienen intereses diversos. El Estado democrático debe respetar el derecho a ser diferentes, asumir la conflictivilidad que esos intereses diversos $-\mathrm{y}$ a veces contrapuestos - representan, reconocer a todos el derecho de expresar sus visiones y a organizarse para buscar la realización de sus objetivos legítimos. El Estado democrático requiere, por

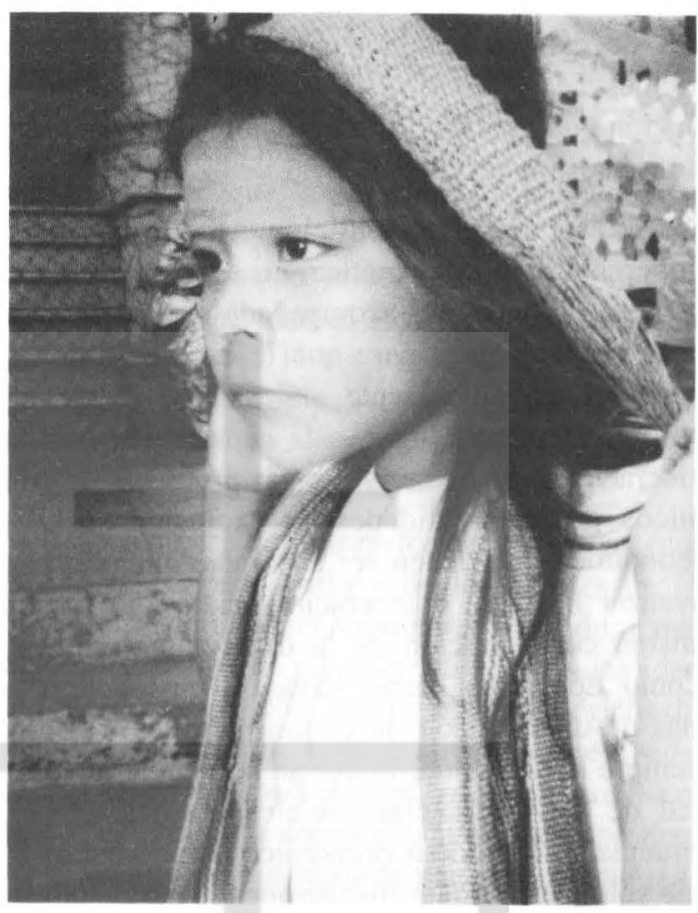

consiguiente, el establecimiento de reglas claras, de consenso de toda la sociedad, para la solución pacífica y participativa de las contradicciones entre los diferentes grupos sociales, permitiendo compromisos entre ellos sobre los niveles y las formas de realización de sus objetivos específicos.

Los acuerdos de Chapultepec y su cumplimiento posterior han posibilitado que se den pasos nada despreciables en la generación de las instituciones indispensables para la democracia. La apertura de espacios de participación legal a grupos políticos antes excluidos es un avance significativo y el proceso electoral de 1994, con todas sus imperfecciones, se constituyó en otra etapa del proceso. La legitimidad democrática, condición necesaria para la gabernabilidad democrática, implica un consenso expresado, explícito, a través de los procesos electorales. Estos son, en última instancia, una suma de las voluntades individuales sobre quiénes deben gobernar el país, expresadas en un momento determinado. Para que las elecciones sean democráticas, se requiere una estructura de poder democrático, así como ésta se consolida con elecciones democráticas. Es decir, para que los comicios sean expresión real de los ciudada- 
nos, se requiere la existencia de ciudadanos capaces de expresarse sin temor a represalias, conscientes de su deber y su derecho a votar por la opción que más favorece sus intereses, y decididos a respetar los resultados.

Si ésta es una condición necesaria para la gobernabilidad democrática, no es condición suficiente. Ella debe ser acompañada de la existencia de otras realidades para que la capacidad de gobernar democráticamente sea estable. Lo que nos remite, en primer lugar, a la capacidad de la democracia política para enfrentar problemas económicos graves, realidades de exclusión social y económica que deben ser superadas no sólo para avanzar hacia la democracia social, sino para la misma consolidación de la democracia política6. Como Edelberto Torres Rivas lo ha repetido en más de una ocasión, la cultura autoritaria "busca siempre la exclusión, lo que se basa en la legitimidad de las diferencias sociales, cuya existencia refuerza". El modelo económico actual, continúa, "no sólo descubre las distinciones sociales sino las profundiza, ...y con ello vuelve crítica la legitimidad de una democracia de ciudadanos desiguales". Establecer democracias políticas en sociedades sin justicia social, dice Torres Rivas, es ahora más urgente que nunca y pareciera ser que aquélla puede contribuir a hacer avanzar en la segunda, en tanto que una mayor justicia social permitirá consolidar la democracia política?.

La participación ciudadana, para la cual se abren canales institucionales y jurídicos, no se desarrolla de acuerdo a las expectativas. Permítaseme mencionar, en primer lugar, que los acuerdos de paz — su cumplimiento y supervisión- estuvieron lejos de convertirse en un problema de la sociedad en su conjunto. Si la guerra en sus últimos días perdía soporte popular en ambos lados, la construcción de la paz se dejó en gran medida a la acción de los contendientes y la supervisión de ONUSAL, la misión de Naciones Unidas para vigilar el proceso. En gran medida, los partidos políticos opositores y no pocas de las fuerzas sociales confiaron en que la existencia de un organismo externo, internacionalizado como parte del aparato de Estado salvadoreños, garantizaba la construcción de las condiciones para la de- mocratización, con todos sus componentes de libertad de participación, cese de la impunidad, desmantelamiento de las instituciones y organizaciones del autoritarismo, etc.

Las elecciones generales de 1994, llamadas por algunos "las elecciones del siglo", nos dan un termómetro de la participación ciudadana. Primero, la baja participación de votantes en unos comicios planteados con tanto dramatismo por el gobierno, los partidos y los observadores y los actores externos; con presiones indecibles para obtener el carnet electoral, aunque con actitudes organizativas negativas, con llamados a ejercer un voto que podía cambiar la historia del país, sólo la mitad de los electores inscritos y algo menos de los que están en capacidad para hacerlo, ejercieron su derecho de elección.

Los comicios estuvieron lejos de tener la cristalinidad necesaria como para dar credibilidad al conjunto de la sociedad. Las llamadas "irregularidades", si bien no puede demostrarse que alteraron sustancialmente los resultados a nivel nacional, pusieron un velo de duda sobre cuánto se había avanzado y se podía avanzar en la construcción de la democracia electoral. $Y$, pese a que muy pocos comparten nuestra opinión, nos parece altamente significativo que la oposición apelara a Naciones Unidas y al senado de Estados Unidos para corregir esas "irregularidades" en la segunda vuelta de la elección presidencial, en vez de demandar la movilización popular para defender la libertad del voto y los resultados de diversos municipios en los cuales el voto se manipuló claramente.

Sin embargo, pese a los problemas descritos, las elecciones han sido la puerta de entrada a una situación de "normalidad" jurídico institucional que cierra la etapa de lo que hemos llamado la "transición pactada" y abre una nueva etapa en el proceso político del país.

Conviene entonces mencionar lo que a nuestro juicio constituyen los signos más preocupantes hacia el futuro.

En primer lugar, la democracia, hemos dicho arriba, la concebimos como un proceso continuo de confrontación para el compromiso. Si el 
autoritarismo y la guerra revolucionaria son por definición confrontación para la liquidación y la ruptura, la democracia está muy lejos de ser en esencia una vida armónica, sin disensos. Hasta hoy, las fuerzas políticas antes revolucionarias han actuado con un criterio desmovilizador, tratando de consensuar todas las decisiones políticas, calificando de desestabilizadora toda confrontación seria a partir de intereses claramente definidos. A esto ayuda la poca claridad conceptual de todos los partidos, ARENA incluida, que no permite distinguir ni qué intereses defienden prioritariamente, ni cuáles son las fronteras de lo que es negociable tácitamente y de lo que exige — ¡si es posible!una negociación estratégica.

En segundo lugar, depositar en la "sociedad civil" la solución de los conflictos es una nueva tentación. Para la izquierda este término parece haber sustituido al antiguo proletariado $o$ al de pueblo. $Y$ hoy, tanto la derecha -jorganismos internacionales incluidos!- como la izquierda dan la impresión de concebir a las organizaciones no gubernamentales como la vanguardia conductora de la sociedad civil. Esto responde a dos concepciones contrarias a la democratización política: (1) que las soluciones a los problemas sociales y políticos son técnicas y unívocas, por lo que la disputa partidaria a nivel electoral se da entre personas plebiscitadas y no entre concepciones; y (2) que lo local es el espacio único para la participación de la sociedad civil y que los canales para ella son las organizaciones no gubernamentales.

En tercer lugar, la aceptación indiscutida de que el "mercado" resuelve los problemas económicos, y por el derrame, los sociales, pone en duda la posibilidad de una democracia aun a sólo nivel político. ¿Es compatible ésta con un programa económico que concentra crecientemente la riqueza, que exige cada día más sujeción de los trabajadores a los intereses del capital, que profundiza las divisiones y dependencias sociales? ¿Es posible que haya democracia si los únicos conflictos que debemos aceptar son los de los intereses de los diversos grupos de la burguesía y la salida de la "izquierda" es optar entre ellos sin considerar los intereses de las clases subalternas?

\section{Conclusiones}

Lo dicho no debe interpretarse como una expresión de pesimismo. Concebir los obstáculos es la primera condición para definir la estrategia del combate. Creo que la oportunidad para entrar en un estadio de mayor democratización no está aún perdida, pese a los nubarrones resultado de nuestra historia de autoritarismo. Y ya hay signos de lucha social, que inician la construcción de un espacio de debate más participativo, que pondrá a prueba la tolerancia del nuevo sistema. Sólo la lucha social favorece la distribución, no el mercado.

Ello requiere de la transformación de las fuerzas sociales. Pero también de los aportes de los cientistas sociales (economistas incluidos) que, desembarazados del complejo del fin de las ideologías, enfrenten con sus principios teóricos las nuevas realidades de la globalización, que ayuden a clarificar los espacios -por limitados que seanen los que la lucha social debe ser dada con decisión, dentro de los cauces de una democracia política. Al fin y al cabo, ésta es un arte del compromiso, sin abandonar los intereses fundamentales.

No pensamos haber hecho aportes teóricos, quizá ni siquiera hemos planteado preguntas trascendentales para la investigación. Como dije al comienzo, ésta es más una intervención para trasladar reflexiones que provienen de una actitud ciudadana, a partir de una larga lucha por la vivencia de la democracia con justicia social.

\section{Notas}

1. Esta sección está tomada de nuestro trabajo "La transición salvadoreña y los partidos políticos", publicado en el Cuaderno de Trabajo No. 6 de FLACSO - Proyecto El Salvador, julio de 1994.

2. Citas tomadas de los textos de los discursos pronunciados en la ceremonia de suscripción de los acuerdos de paz en el Palacio de Chapultepec, México, D.F., 16 de enero de 1992.

3. FLACSO - Proyecto El Salvador, "A dos años de la firma de los Acuerdos de paz", Cuaderno de Trabajo $N^{\circ}$ 5, San Salvador, 11 de enero de 1994, p. 14.

4. Ya anteriormente, el Frente Democrático Revolucionario, convertido en Convergencia Democrática, se había incorporado a la vida política legal, y 
Guillermo Manuel Ungo estaba inscrito como candidato presidencial. Sin embargo, no había una declaración expresa del FMLN aceptando la disputa electoral y la legalidad existentes.

5. Estas ideas las expresamos ya en nuestro trabajo "Gobernabilidad, elecciones y democracia", Cuaderno de Trabajo No. 6, FLACSO - Programa El
Salvador, julio de 1994.

6. Ver Francisco C. Weffort, ¿Cuál democracia?, FLACSO, San José, Costa Rica, 1993.

7. Ver, entre otros escritos de Torres Rivas, la "Presentación" al libro de Weffort citado anteriormente.

8. Creo que este es un problema teórico-práctico poco estudiado por los cientistas políticos. 